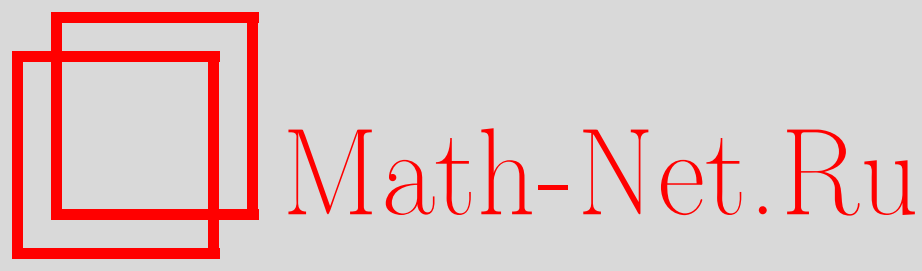

С. В. Талалов, Решения типа струн, вихрей и энионов для иерархии нелинейного уравнения Шредингера, TMФ, 2013, том 176, номер 3, 372-384

DOI: https://doi.org/10.4213/tmf8466

Использование Общероссийского математического портала Math-Net.Ru подразумевает, что вы прочитали и согласны с пользовательским соглашением http://www . mathnet.ru/rus/agreement

Параметры загрузки:

IP: 35.174 .16 .151

26 апреля 2023 г., 17:37:05






\title{
ФИЗИКА
}

Том 176, № 3

сентябрь, 2013

\section{РЕШЕНИЯ ТИПА СТРУН, ВИХРЕЙ И ЭНИОНОВ ДЛЯ ИЕРАРХИИ НЕЛИНЕЙНОГО УРАВНЕНИЯ ШРЕДИНГЕРА}

\begin{abstract}
Рассмотрено отображение, сопоставляющее каждому уравнению иерархии нелинейного уравнения Шредингера и модифицированного уравнения Кортевега-де Фриза бесконечную кривую, эволюционирующую в пространстве $E_{3}$. Показано, что первые ступени иерархии соответствуют известным объектам: струнам и вихревым нитям с различной структурой. Подробно рассмотрена одна из ступеней иерархии, отвечающая динамике вихревой нити в приближении локальной индукции. Построено гамильтоново описание соответствующей динамики, допускающее интерпретацию в терминах квазичастицы на плоскости "эниона". Предложена схема квантования теории, в рамках которой получена формула для (вообще говоря, дробного) значения спина.
\end{abstract}

Ключевые слова: иерархия нелинейного уравнения Шредингера, модифицированное уравнение Кортевега-де Фриза, вихри, энионы.

DOI: $10.4213 / \operatorname{tmf} 8466$

\section{1. ВВЕДЕНИЕ}

Несмотря на несомненный успех Стандартной Модели, попытки представить наблюдаемые частицы как коллективные возбуждения некоторой первичной фундаментальной среды по-прежнему появляются в литературе [1]. Наиболее известной как в историческом, так и, по-видимому, в эстетическом плане является идея лорда Кельвина (Томсона), согласно которой атомы представляют собой заузленные вихревые трубки эфира [2]. Хотя данная теория и не получила детального развития, высказанные Томсоном гипотезы продолжают вызывать значительный интерес среди исследователей [3]. Между тем такой подход - представление "частицы" как некоторого коллективного возбуждения бесконечномерной динамической ситемы является на сегодняшний день распространенным при описании частиц с дробной статистикой - энионов [4]. Ключевым моментом при построении моделей энионов является неодносвязность множества $\mathcal{X} \backslash\left[\boldsymbol{z}_{i}\right]$, где $\mathcal{X}$ - пространство координат рассматриваемых объектов (частиц), а $\left[\boldsymbol{z}_{i}\right]$ - совокупность значений этих координат

*Тольяттинский государственный университет, Тольятти, Самарская обл., Россия. E-mail: svtalalov@tltsu.ru 
в фиксированный момент времени. В этой связи представляется естественным, что теория изначально строится как планарная (см., например, [5], [6]). Вместе с этим нетривиальные гомотопические группы возникают, очевидно, и в теориях, в которых координаты объектов заданы в пространстве $E_{3}$. Примером могут служить теории, описывающие бесконечные (в том числе космические) струны, протяженные одномерные дефекты в конденсированных средах, вихревые нити и т. п. Известно, что при взаимодействии "обычных" частиц с подобными объектами возможно появление дробной статистики [7]. В работе [8] был предложен пример несколько иного рода: свободная бесконечная струна, эволюционирующая в пространстве $E_{3}$, была описана в терминах фазового пространства, симплектические листы которого имеют вид $\mathcal{H}_{2} \times \mathcal{H}_{0}$. Здесь $\mathcal{H}_{2}$ - фазовое пространство свободной частицы на плоскости, а пространство $\mathcal{H}_{0}$ бесконечномерно и имеет фундаментальные координаты, инвариантные относительно группы $E(2)$. В теории также имеется связь, перепутывающая "внешние" $\left(\mathcal{H}_{2}\right)$ и "внутренние" $\left(\mathcal{H}_{0}\right)$ степени свободы. В работе [9] была анонсирована возможность сопоставления определенным образом каждому уравнению иерархии нелинейного уравнения Шредингера и модифицированного уравнения Кортевега-де Фриза (НШ-мКдФ) некоторой бесконечной эволюционирующей кривой; в статьях [8], [9] рассматривался случай, когда такая кривая является релятивистской струной. Цель настоящей работы - построить анонсированное отображение явно и исследовать некоторые высшие (по отношению к струне) ступени иерархии.

Пусть $\mathcal{C}\left(E_{3}\right)$ - класс гладких почти всюду (за исключением, быть может, конечного числа точек возврата) бесконечных кривых $\mathbf{z}(\xi)$ в пространстве $E_{3}$ вида

$$
\mathbf{z}(\xi)=\mathbf{z}_{0}+\gamma(\xi)+\mathbf{a} \sqrt{\frac{2}{\pi}} \int_{0}^{\xi} e^{-\eta^{2}} d \eta+\xi \mathbf{n}, \quad-\infty<\xi<\infty
$$

где постоянные векторы $\mathbf{z}_{0}, \mathbf{a}, \mathbf{n}$ - произвольны $(\mathbf{n} \neq 0)$, а компоненты вектор-функции $\gamma(\xi)$ убывают при $|\xi| \rightarrow \infty$ быстрее любой степени безразмерного параметра $\xi$. $\mathrm{B}$ дальнейшем для любых векторов $\mathbf{x} \in E_{3}$ там, где это удобно, будет использоваться матричное представление $X=\sum_{i=1}^{3} x_{i} \boldsymbol{\sigma}_{i}$, где $\boldsymbol{\sigma}_{i}$ - стандартные матрицы Паули, а $x_{i}$ - координаты в некотором ортонормированном базисе $\mathbf{b}_{i}$. Далее, пусть $\rho(\xi)-$ комплекснозначная функция из пространства Шварца $\mathcal{S}\left(\mathbb{R}_{1}\right), \mathbf{z}_{0}$ - произвольный вектор пространства $E_{3}$, а $\alpha_{0}$ - некоторый фиксированный параметр с размерностью длины. Рассмотрим отображение

$$
\mathcal{S}\left(\mathbb{R}_{1}\right) \supset \mathcal{S}_{0} \rightarrow \mathcal{C}\left(E_{3}\right): \rho(\xi) \rightarrow Z(\xi)=Z_{0}+\frac{\alpha_{0}}{2}-\int_{-\infty}^{\infty} \varepsilon(\xi-\eta) T^{+}(\eta) \boldsymbol{\sigma}_{3} T(\eta) d \eta,
$$

где $T(\xi) \in S U(2)$ - матрица решений вспомогательной линейной системы первого порядка

$$
\partial_{\xi} T(\xi)+Q(\xi) T(\xi)=0
$$

Матрица коэффициентов здесь имеет вид $Q(\xi)=-\rho(\xi) \boldsymbol{\sigma}_{-}+\bar{\rho}(\xi) \boldsymbol{\sigma}_{+}$, а подмножество $\mathcal{S}_{0} \subset \mathcal{S}\left(\mathbb{R}_{1}\right)$ определено требованием

$$
\mathfrak{M}=\epsilon \mathbf{1}_{2}, \quad \epsilon= \pm 1
$$


где $\mathfrak{M}=\mathfrak{M}[\rho]-$ матрица монодромии системы (3). В дальнейшем будет полезным представление

$$
T(\xi)=T_{0}(\xi) U, \quad \lim _{\xi \rightarrow-\infty} T_{0}(\xi)=\mathbf{1}_{2},
$$

где постоянная матрица $U \in S U(2)$ связана с вектором $\mathbf{n}$ формулой

$$
N=\left(\frac{\alpha_{0}}{2}\right) U^{+} \boldsymbol{\sigma}_{3} U .
$$

В работах [8], [9], детально исследовались как мотивировка, так и структура заявленного отображения в случае, когда исходным объектом при рассмотрении кривых указанного класса были релятивистские струны. После выполнения нерелятивистской редукции струна имела представление вида $\mathbf{z}=\mathbf{z}_{+}+\mathbf{z}_{-}$, где векторы $\mathbf{z}_{+}$и $\mathbf{z}_{-}$ строились по формулам (2) для функций $\rho_{+}$и $\rho_{-}$соответственно. При этом стандартная струнная динамика (порожденная действием Намбу-Гото) в ортонормальной параметризации приводила к эволюционным уравнениям для функций $\rho_{ \pm}$:

$$
\partial_{\mp} \rho_{ \pm}=0, \quad \partial_{ \pm}=\frac{\partial}{\partial \xi_{ \pm}}, \quad \xi_{ \pm}=\xi \pm \tau .
$$

Рассмотрим, например, уравнение $\partial_{+} \rho_{-}=0$. Данное уравнение может быть рассмотрено как уравнение с номером $n=2$ известной иерархии НШ-мКдФ [10]. Как было замечено в работе [9], распространение соответствия (2) на все ступени указанной иерархии приводит к некоторой иерархии динамик бесконечных кривых в пространстве $E_{3}$. В настоящей работе мы исследуем случаи динамик при $n \neq 2$ и продемонстрируем естественность такого обобщения.

\section{2. ИЕРАРХИЯ КРИВЫХ ПРИ $n \neq 2$}

Иерархия НШ-мКдФ может быть получена [11], например, как условие совместности линейных систем

$$
\begin{aligned}
\partial_{\xi} T(\xi) & =U(\xi ; \lambda) T(\xi), \\
\partial_{\tau} T(\xi) & =V_{n}(\xi ; \lambda) T(\xi) .
\end{aligned}
$$

В наших обозначениях $U(\xi ; \lambda)=-Q(\xi)+i \lambda \boldsymbol{\sigma}_{3}$, зависимость от параметра эволюции $\tau$ мы, по возможности, явно не указываем. Алгоритм построения полиномиальных (по параметру $\lambda$ ) матриц $V_{n}$ детально прописан в книге [11]. Так, например, $V_{2}(\xi ; 0)=Q(\xi)$. Переходя здесь к безразмерным координатам $\mathbf{z}(\xi) \rightarrow \mathbf{z}(\xi) / \alpha_{0}$, исследуем в качестве примеров некоторые ступени иерархии при $n \neq 2$.

Рассмотрим простейший случай $n=1$. При этом $V_{1}=(i / 2) \boldsymbol{\sigma}_{3}$, так что в соответствии с определением (2) и системой (8) для вектор-функции $\mathbf{z}(\xi)=\mathbf{z}(\tau, \xi)$ имеем $\partial_{\tau} \mathbf{z}(\xi)=0$. Таким образом, первая ступень рассматриваемой иерархии отвечает стационарным кривым класса $\mathcal{C}\left(E_{3}\right)$.

Рассмотрим теперь случай $n=3$, отвечающий собственно нелинейному уравнению Шредингера. Для матрицы $V_{3}(\xi)=V_{3}(\xi, 0)$ имеем ${ }^{1)}$

$$
V_{3}(\xi)=-i\left(\partial_{\xi} Q(\xi)-Q^{2}(\xi)\right) \boldsymbol{\sigma}_{3}
$$

\footnotetext{
1) В настоящей работе установлено такое соответствие с обозначениями [11]: $\rho \equiv \rho_{-} \leftrightarrow i \psi$.
} 
так что для $Z(\xi)$ находим

$$
\begin{aligned}
\partial_{\xi} Z(\xi) & =T^{+}(\xi) \boldsymbol{\sigma}_{3} T(\xi), \\
\partial_{\tau} Z(\xi) & =2 i T^{+}(\xi) Q(\xi) T(\xi) .
\end{aligned}
$$

Как прямое следствие уравнений $(3),(9),(10)$ для функции $\mathbf{z}(\tau, \xi)$ получаем

$$
\partial_{\tau} \mathbf{z}(\tau, \xi)=\partial_{\xi} \mathbf{z}(\tau, \xi) \times \partial_{\xi}^{2} \mathbf{z}(\tau, \xi) .
$$

Таким образом, в случае $n=3$ кривая (2) - это вихревая нить в приближении “локальной индукции" ${ }^{2)}$. Здесь необходимо заметить, что факт появления нелинейного уравнения Шредингера при описании такого объекта известен давно (см., например, обсуждение в книге [13]).

Пусть теперь $n=4$. Динамическое уравнение для функции $\rho(\tau, \xi)$ в этом случае есть модифицированное уравнение Кортевега-де Фриза. В соответствии с алгоритмом [11] для матрицы $V_{4}(\xi)$ имеем

$$
V_{4}(\xi)=-\partial_{\xi}^{2} Q(\xi)+Q(\xi)\left(\partial_{\xi} Q(\xi)\right)-\left(\partial_{\xi} Q(\xi)\right) Q(\xi)-2|\rho(\xi)|^{2} Q(\xi) .
$$

Используя формулы (7)-(10), убеждаемся в справедливости следующих выражений:

$$
\begin{aligned}
& \partial_{\xi}^{3} Z(\xi)=\partial_{\xi}^{2}\left(T^{+}(\xi) \boldsymbol{\sigma}_{3} T(\xi)\right)=-2 T^{+}(\xi) \boldsymbol{\sigma}_{3}\left(\partial_{\xi} Q(\xi)\right) T(\xi)-4|\rho(\xi)|^{2} T^{+}(\xi) \boldsymbol{\sigma}_{3} T(\xi), \\
& \partial_{\tau} Z(\xi)=-2 T^{+}(\xi) \boldsymbol{\sigma}_{3}\left(\partial_{\xi} Q(\xi)\right) T(\xi)+2|\rho(\xi)|^{2} T^{+}(\xi) \boldsymbol{\sigma}_{3} T(\xi) .
\end{aligned}
$$

Отсюда следует равенство

$$
\partial_{\tau} Z(\xi)=\partial_{\xi}^{2}\left(T^{+}(\xi) \boldsymbol{\sigma}_{3} T(\xi)\right)+6|\rho(\xi)|^{2} T^{+}(\xi) \boldsymbol{\sigma}_{3} T(\xi) .
$$

\section{Поскольку}

$$
\left|\partial_{\xi}^{2} \mathbf{z}(\xi)\right|^{2}=\frac{1}{2} \operatorname{Tr}\left(\partial_{\xi}\left(T^{+}(\xi) \boldsymbol{\sigma}_{3} T(\xi)\right) \partial_{\xi}\left(T^{+}(\xi) \boldsymbol{\sigma}_{3} T(\xi)\right)\right)=8|\rho(\xi)|^{2},
$$

эволюционное уравнение для кривой $\mathbf{z}(\tau, \xi)$ принимает вид

$$
\partial_{\tau} \mathbf{z}(\tau, \xi)=\partial_{\xi}^{3} \mathbf{z}(\tau, \xi)+\frac{3}{2}\left|\partial_{\xi}^{2} \mathbf{z}(\tau, \xi)\right|^{2} \partial_{\xi} \mathbf{z}(\tau, \xi)
$$

Данное уравнение совпадает с обобщением локально-индукционного уравнения (11) при наличии в ядре вихря некоторой аксиальной скорости течения $w_{\mathrm{a}}-$ в асимптотическом пределе $w_{\mathrm{a}} \rightarrow \infty$ (см. [13]). Таким образом, рассмотренные выше примеры демонстрируют естественность сопоставления с помощью формулы (2) уравнениям иерархии НШ-мКдФ эволюционирующих кривых класса $\mathcal{C}\left(E_{3}\right)$. Установлено соответствие:

$[n=1] \leftrightarrow$ стационарные кривые;

$[n=2] \leftrightarrow$ свободные струны;

2) Данный термин в гидродинамике означает приближение, в котором движение всякой точки вихревой нити является следствием воздействия на нее не всего вихря, а только его части окрестности данной точки размером порядка радиуса кривизны нити (в данной точке) [12], [13]. 
$[n=3] \leftrightarrow$ вихревые нити в приближении локальной индукции (бесструктурное ядро);

$[n=4] \leftrightarrow$ вихревые нити в приближении локальной индукции (ядро с внутренней структурой, $\left.w_{\mathrm{a}} \rightarrow \infty\right)$.

Будучи связанными иерархией НШ-мКдФ единым образом, данные динамические системы (конечно, давно изученные разными авторами и в разное время) делают естественными и "высшие" кривые, которые при $n>4$ являются уже новыми объектами. Так, при $n=5$ для кривой получаем эволюционное уравнение

$$
\partial_{\tau} \mathbf{z}(\tau, \xi)=\partial_{\xi} \mathbf{z}(\tau, \xi) \times\left[\partial_{\xi}^{4} \mathbf{z}(\tau, \xi)+\frac{3}{2}\left|\partial_{\xi}^{2} \mathbf{z}(\tau, \xi)\right|^{2} \partial_{\xi}^{2} \mathbf{z}(\tau, \xi)\right] .
$$

\section{3. ДИНАМИЧЕСКИЕ ИНВАРИАНТЫ ВИХРЕВОЙ НИТИ}

Далее мы рассмотрим более детально ступень $n=3$ иерархии, которая, как показано выше, отвечает бесконечной вихревой нити в приближении локальной индукции. При этом будем следовать схеме, реализованной в работе [8] для случая $n=2$. В дальнейшем для упрощения формул базис $\mathbf{b}_{i}$ мы выбираем так, что $\mathbf{b}_{3} \propto \mathbf{n}$. Стандартные выражения для вихревого импульса и вихревого момента [14]

$$
\mathbf{p}_{\text {canon }}=\varrho \int \mathbf{r} \times \boldsymbol{\omega}(\mathbf{r}) d V, \quad \mathbf{j}_{\text {canon }}=\frac{\varrho}{3} \int \mathbf{r} \times(\mathbf{r} \times \boldsymbol{\omega}(\mathbf{r})) d V
$$

предполагают, что “завихренность" $\boldsymbol{\omega}(\mathbf{r})$ убывает достаточно быстро при $r \rightarrow \infty$. В нашем случае это не так, поэтому интегралы в правых частях (14), вообще говоря, расходятся. Действительно, для бесконечной вихревой нити имеем [13]

$$
\boldsymbol{\omega}(\mathbf{r})=\Gamma \lim _{R \rightarrow \infty} \int_{-R}^{R} \delta(\mathbf{r}-\mathbf{z}(\xi)) \partial_{\xi} \mathbf{z}(\xi) d \xi
$$

Константа $\varrho$ в формулах (14) имеет смысл “плотности жидкости", величина Г смысл "циркуляции". Поскольку далее указанные константы встречаются только в виде произведения, мы везде рассматриваем величину $\Gamma_{\varrho}=\varrho \Gamma$. В нашем случае данная величина является дополнительной динамической переменной теории, определяющей “интенсивность” вихря ${ }^{3)}$. Таким образом, формально канонические инварианты (14) суть пределы при $R \rightarrow \infty$ величин

$$
\mathbf{p}(R)=\Gamma_{\varrho} \int_{-R}^{R} \boldsymbol{\pi}(\xi) d \xi, \quad \mathbf{j}(R)=\frac{\Gamma_{\varrho}}{3} \int_{-R}^{R} \iota(\xi) d \xi
$$

где $\boldsymbol{\pi}(\xi)=\mathbf{z}(\tau, \xi) \times \partial_{\xi} \mathbf{z}(\tau, \xi)$ и $\boldsymbol{\iota}(\xi)=\mathbf{z}(\tau, \xi) \times\left(\mathbf{z}(\tau, \xi) \times \partial_{\xi} \mathbf{z}(\tau, \xi)\right)$.

При $\xi \rightarrow \pm \infty$ справедливо $\mathbf{z}(\xi) \longrightarrow \mathbf{z}_{0} \pm \mathbf{a}+\xi \mathbf{b}_{3}+\mathcal{O}\left(\xi^{-\infty}\right)$, так что выполняется

$$
\pi_{i}(\xi) \in \mathrm{C}(\mathbb{R}), \quad i=1,2,3 ; \quad \iota_{3}(\xi) \in \mathrm{C}(\mathbb{R}) ; \quad \iota_{i}(\xi) \notin \mathrm{C}(\mathbb{R}) \quad i=1,2,
$$

3)Этот факт - так же, как и написание слов "завихренность" и т. д. в кавычках - связан с тем, что движение жидкости в пространстве не является исходным пунктом нашей модели: формулы (11) и (14) рассматриваются как независимые и дополняющие друг друга. 
где $\mathrm{C}(\mathbb{R})$ - класс ограниченных функций. Поэтому компоненты вектора $\mathbf{p}(R)$, так же как и величина $j_{3}(R)$, расходятся линейно при $R \rightarrow \infty$, а величины $j_{1}(R)$ и $j_{2}(R)$ при $\mathbf{a} \neq 0$ расходятся, вообще говоря, квадратично. Заметим, что существуют пределы

$$
\lim _{R \rightarrow \infty} \partial_{\tau} \mathbf{p}(R)=0, \quad \lim _{R \rightarrow \infty} \partial_{\tau} \mathbf{j}(R)=6 \Gamma_{\varrho}\left(\mathbf{a} \times \mathbf{b}_{3}\right) .
$$

Последнее выражение показывает, что определение компонент момента $j_{1}$ и $j_{2}$ как динамических инвариантов при помощи канонических выражений (14) является проблематичным даже с учетом возможной регуляризации интегралов.

Рассмотрим сначала частный случай теории, налагая на класс кривых (1) условие $\mathbf{a}=0$. Тогда соответствующую эволюционирующую кривую можно рассматривать как локальное возмущение бесконечной прямолинейной вихревой нити, параллельной оси $\mathbf{b}_{3}$ и проходящей через точку с координатами $\mathbf{z}_{0}$. Расходимости всех компонент векторов $\mathbf{p}(R)$ и $\mathbf{j}(R)$ в этом случае линейные. С учетом асимптотик радиус-вектора $\mathbf{z}(\xi)$ при больших $R$ находим

$$
\mathbf{p}(R) \simeq \Gamma_{\varrho} R\left(\mathbf{z}_{0} \times \mathbf{b}_{3}\right)+\mathbf{p}_{0}(R)
$$

где

$$
\mathbf{p}_{0}(R)=\frac{\Gamma_{\varrho}}{4} \iint_{-R}^{R} \varepsilon(\xi-\eta) \partial_{\eta} \mathbf{z}(\tau, \eta) \times \partial_{\xi} \mathbf{z}(\tau, \xi) d \xi d \eta .
$$

Отметим, что правая часть (17) сходится при $R \rightarrow \infty$. Соответствующий предел (который не зависит от времени $\tau$ и является трансляционно-инвариантным) мы по определению будем считать импульсом рассматриваемой динамической системы:

$$
\mathbf{p}=\lim _{R \rightarrow \infty} \mathbf{p}_{0}(R) \equiv \alpha_{0}^{2} \Gamma_{\varrho} \boldsymbol{f}_{p}[\rho ; U]
$$

Здесь введено обозначение для вектора $\boldsymbol{f}_{p}$; в матричной форме

$$
F_{p}=\frac{1}{4} \iint_{-\infty}^{\infty} \varepsilon(\xi-\eta) T^{+}(\eta) \boldsymbol{\sigma}_{3} T(\eta) T^{+}(\xi) \boldsymbol{\sigma}_{3} T(\xi) d \xi d \eta
$$

Для момента принимаем аналогичное определение:

$$
\mathbf{j}=\lim _{R \rightarrow \infty} \mathbf{j}_{0}(R)
$$

где

$$
\mathbf{j}_{0}(R)=\frac{\Gamma_{\varrho}}{12} \iiint_{-R}^{R} l_{R}\left(\eta_{1}, \eta_{2}, \eta_{3}\right) \partial_{\eta_{1}} \mathbf{z}\left(\tau, \eta_{1}\right) \times\left(\partial_{\eta_{2}} \mathbf{z}\left(\tau, \eta_{2}\right) \times \partial_{\eta_{3}}^{2} \mathbf{z}\left(\tau, \eta_{3}\right)\right) d \eta_{1} d \eta_{2} d \eta_{3} .
$$

Заметим, что для функции

$$
l_{R}\left(\eta_{1}, \eta_{2}, \eta_{3}\right) \equiv \frac{1}{2} \int_{-R}^{R} \varepsilon\left(\xi-\eta_{1}\right) \varepsilon\left(\xi-\eta_{2}\right) \varepsilon\left(\xi-\eta_{3}\right) d \xi
$$

при $R>\max \left\{\left|\eta_{1}\right|,\left|\eta_{2}\right|,\left|\eta_{3}\right|\right\}$ справедливо

$$
l_{R}\left(\eta_{1}, \eta_{2}, \eta_{3}\right)=\sum_{i=1}^{3} \varepsilon_{i} \eta_{i} \equiv l\left(\eta_{1}, \eta_{2}, \eta_{3}\right), \quad \varepsilon_{i}=\varepsilon_{k}=-1, \quad \varepsilon_{j}=1, \quad \eta_{i}<\eta_{j}<\eta_{k} .
$$


Как и компоненты вектора $\mathbf{p}$, компоненты $\mathbf{j}$ не зависят от времени и трансляционноинвариантны. Смысл данных определений - вычитание из канонических выражений (14) соответственно импульса и момента бесконечной прямолинейной вихревой нити, параллельной оси $\mathbf{b}_{3}$ и проходящей через точку с координатами $\mathbf{z}_{0}$. Таким образом, определяемые импульс и момент - это импульс и момент возмущений исходного (прямолинейного) вихря. Заметим, что идея подобного вычитания, устраняющего расходимости интегралов на бесконечности, для плоских течений была высказана еще в книге [14].

Рассмотрим теперь общий случай $\mathbf{a} \neq 0$. Здесь, как и при $\mathbf{a}=0$, допустима лишь та или иная линейная регуляризация, поскольку требование конечной линейной плотности величин (14) представляется вполне естественным. При определении пуассоновой структуры теории тем или иным способом предполагается, что лиевы операторы, отвечающие динамическим инвариантам $\mathbf{p}, \mathbf{j}, \ldots$, являются генераторами трансляций, поворотов и т.д. Однако в силу сказанного выше при любой линейной регуляризации по-прежнему $j_{1}(R) \rightarrow \infty$ и $j_{2}(R) \rightarrow \infty$ при $R \rightarrow \infty$. Ситуация, когда два $\left(j_{1}\right.$ и $\left.j_{2}\right)$ из трех генераторов поворотов группы $S O(3)$ формально (например, введением дополнительного параметра) устремляются к бесконечности, соответствует контракции [15]

$$
S O(3) \longrightarrow E(2)
$$

где $E(2)$ - группа движений плоскости $E_{2} \perp \mathbf{b}_{3}$. При этом сами величины $j_{1}$ и $j_{2}$ в некотором смысле переходят в генераторы трансляций в плоскости $E_{2}$. В нашем случае генераторы таких трансляций уже имеются - это величины $p_{1}$ и $p_{2}$. Поэтому возникающая в общем случае $\mathbf{a} \neq 0$ "естественная" контракция группы $S O(3)$ делает определение величин $j_{1}$ и $j_{2}$ избыточным. Поскольку исходной группой пространственно-временно́й симметрии теории является группа $E(3) \times E_{\tau}$, где $E_{\tau}-$ абелева группа временнь́х сдвигов, мы имеем редукцию

$$
E(3) \times E_{\tau} \longrightarrow E(2) \times E_{\xi} \times E_{\tau}
$$

Здесь $E_{\xi}$ - группа сдвигов параметра $\xi$. В соотношении (21) учтен тот факт, что трансляция вида $\mathbf{z}(\tau, \xi) \rightarrow \mathbf{z}(\tau, \xi)+\delta \mathbf{b}_{3}$ эквивалентна сдвигу $\xi \rightarrow \xi+\delta$ совместно с пространственно-инвариантным преобразованием $\rho(\xi) \rightarrow \rho(\xi-\delta)$. После выделения направления $\mathbf{b}_{3}$ и редукции $(21)$ в представлении (5) матриц решений $T(\xi)$ допустимы лишь матрицы $U$ вида $U=\operatorname{diag}\left(e^{i \beta}, e^{-i \beta}\right)$. Параметр $\beta \in[0,2 \pi)$ имеет смысл угла поворота вокруг вектора $\mathbf{b}_{3}$. Таким образом, рассмотрение вихревых нитей общего вида (1) требует редукции группы пространственно-временно́й симметрии теории; далее такая редукция позволит построить гамильтонову динамическую систему, интерпретируемую как квазичастица на плоскости.

Вернемся к вопросу определения динамических инвариантов $\mathbf{p}$ и $s=j_{3}$. Заметим, что правые части (17) и (20) сходятся при $R \rightarrow \infty$ и в общем случае $\mathbf{a} \neq 0$. Поэтому импульс р мы по-прежнему определяем формулой (18), а момент $s$ - формулой

$$
s=\mathbf{b}_{3} \cdot \lim _{R \rightarrow \infty} \mathbf{j}_{0}(R) \equiv \alpha_{0}^{3} \Gamma_{\varrho} f_{s}[\rho]
$$


Здесь, как и при определении р, введено обозначение

$$
\begin{aligned}
f_{s}= & \frac{1}{12} \operatorname{Tr}\left(\boldsymbol{\sigma}_{3} \iiint_{-\infty}^{\infty} l\left(\eta_{1}, \eta_{2}, \eta_{3}\right) T^{+}\left(\eta_{1}\right) \boldsymbol{\sigma}_{3} T\left(\eta_{1}\right) T^{+}\left(\eta_{2}\right) \boldsymbol{\sigma}_{3} T\left(\eta_{2}\right) \times\right. \\
& \left.\times T^{+}\left(\eta_{3}\right) Q\left(\eta_{3}\right) \boldsymbol{\sigma}_{3} T\left(\eta_{3}\right) d \eta_{1} d \eta_{2} d \eta_{3}\right) .
\end{aligned}
$$

Легко видеть, что величина $f_{s}$ от угла $\beta$ не зависит.

\section{4. ВИХРЕВАЯ МОДЕЛЬ КВАЗИЧАСТИЦЫ}

Итак, рассматриваемая динамическая система "бесконечная вихревая нить" полностью определена заданием следующих переменных:

$$
\rho(\xi) \in \mathcal{S}_{0} \subset \mathcal{S}\left(\mathbb{R}_{1}\right) ; \quad \mathbf{z}_{0}=z_{01} \mathbf{b}_{1}+z_{02} \mathbf{b}_{2}, \quad \beta \in[0,2 \pi), \quad \Gamma_{\varrho} .
$$

По данному набору переменных восстанавливаются как координаты точек нити, так и динамические инварианты $\mathbf{p}$ и $s$. Пусть $\mathcal{P}=p_{1} \mathbf{b}_{1}+p_{2} \mathbf{b}_{2}-$ импульс вихревой нити в плоскости $E_{2} \perp \mathbf{b}_{3}$. Тогда

$$
\mathcal{P}=\alpha_{0}^{2} \Gamma_{\varrho} \boldsymbol{f}[\rho, \beta]
$$

где $\boldsymbol{f}=\left(f_{p}\right)_{1} \mathbf{b}_{1}+\left(f_{p}\right)_{2} \mathbf{b}_{2}$. Исключая из формул $(22)$ и (24) динамическую переменную $\Gamma_{\varrho}$, находим

$$
\alpha_{0}|\mathcal{P}| f_{s}[\rho]=s|\boldsymbol{f}[\rho]|
$$

Заметим, что функционал $\boldsymbol{f}^{2}=-\operatorname{det} F_{p}$, в отличие от $\boldsymbol{f}$, от угла $\beta$ не зависит.

Необходимые для восстановления вихревой нити по набору переменных (23) угол $\beta$ и величина $\Gamma_{\varrho}$ могут быть выражены при заданной функции $\rho(\xi)$ через величины $p_{1}$ и $p_{2}$ :

$$
\Gamma_{\varrho}=\alpha_{0}^{-2} \frac{|\mathcal{P}|}{|\boldsymbol{f}|}, \quad \beta=\frac{1}{2}\left[\arg \left(p_{1}+i p_{2}\right)-\arg \left(\left(f_{p}^{0}\right)_{1}+i\left(f_{p}^{0}\right)_{2}\right)\right],
$$

где $\left(f_{p}^{0}\right)_{j}$ - функционалы $\left(f_{p}\right)_{j}$, построенные по элементам матриц $T_{0}($ см. $(5))$. Этот факт позволяет считать в дальнейшем величины $p_{1}$ и $p_{2}$ независимыми динамическими переменными вместо переменных $\beta$ и $\Gamma_{\varrho}$. В число независимых переменных мы включаем также величину $s$ : поскольку она является избыточной, соотношение (25) в этом случае необходимо рассматривать как связь. Естественность включения собственного момента $s$ в число независимых переменных будет обсуждаться ниже.

$\mathrm{B}$ терминах независимых переменных $p_{1}$ и $p_{2}$ представляется естественным кроме пространственно-временнь́х преобразований, допустимых группой $E(2) \times E_{\xi} \times E_{\tau}$, рассматривать также галилеевские бусты

$$
p_{j} \longrightarrow \tilde{p}_{j}=p_{j}+c v_{j}, \quad c, v_{j}=\text { const }, \quad j=1,2 \text {. }
$$

Допущение таких преобразований означает переход к более широкой группе пространственно-временно́й симметрии - группе $\widetilde{\mathcal{G}_{2}} \times E_{\xi}$, где $\widetilde{\mathcal{G}_{2}}-$ группа Галилея плоскости; тильда означает однопараметрическое $\left(m_{0}\right)$ центральное расширение, которое мы будем далее считать выполненным. 
Сделанное расширение группы симметрии позволяет рассмотреть вопрос об энергии системы с групповой точки зрения. Известно, что прямым следствием идеализированных представлений о бесконечной протяженности и нулевом диаметре вихревой нити является бесконечность энергии, рассчитанной с использованием формулы Био-Савара [14]. Поэтому в предлагаемой модели мы постулируем, что внутренняя энергия бесконечной прямолинейной стационарной нити есть $\mathcal{E}_{0}{ }^{4}$ ). Для нахождения полной энергии искривленной нити мы поступим следующим образом. Как известно (см., например, [16]), алгебра группы $\widetilde{\mathcal{G}}_{2}$ имеет три функции Казимира:

$$
\hat{C}_{1}=m_{0} \hat{I}, \quad \hat{C}_{2}=\hat{J}-\sum_{i, j=1}^{2} \epsilon_{j k} \hat{P}_{j} \hat{B}_{k}, \quad \hat{C}_{3}=\hat{H}-\frac{1}{2 m_{0}} \sum_{i=1}^{2} \hat{P}_{i}^{2},
$$

где $\hat{I}$ - единичный оператор, $\hat{J}, \hat{H}, \hat{P}_{i}$ и $\hat{B}_{i}$ - генераторы поворотов, временнь́х и пространственных сдвигов, а также галилеевских бустов соответственно. Функция Казимира $\hat{C}_{3}$ интерпретируется как внутренняя энергия частицы [16]. С учетом этого представляется естественным в качестве энергии рассматриваемой вихревой нити принять выражение

$$
\mathcal{E}=\frac{\mathcal{P}^{2}}{2 m_{0}}+\mathcal{E}_{0}(1+h[\rho]),
$$

где функционал $h[\rho]$ - гамильтониан нелинейного уравнения Шредингера, обеспечивающий динамику “внутренних" (т. е. инвариантных относительно группы $\widetilde{\mathcal{G}}_{2}$ ) степеней свободы $\rho(\xi)$. Его явный вид зависит от пуассоновой структуры теории; так, при скобках Пуассона для переменных $\rho(\xi)$ и $\bar{\rho}(\xi)$, определяющих “вторую гамильтонову структуру" нелинейного уравнения Шредингера, имеем [10], [11]

$$
h[\rho]=-i \int_{-\infty}^{\infty} \bar{\rho}(\xi) \partial_{\xi} \rho(\xi) d \xi
$$

Далее вместо величин $z_{01}$ и $z_{02}$ мы будем использовать переменные

$$
q_{i}=m_{0} z_{0 i}-\tau t_{0} p_{i}, \quad t_{0}=\sqrt{\frac{m_{0} \alpha_{0}^{2}}{\mathcal{E}_{0}}}, \quad i=1,2 .
$$

Таким образом, фундаментальными переменными теории объявляются величины

$$
\rho(\xi) \in \mathcal{S}_{0} \subset \mathcal{S}\left(\mathbb{R}_{1}\right), \quad \boldsymbol{q}=q_{1} \mathbf{b}_{1}+q_{2} \mathbf{b}_{2}, \quad \mathcal{P}=p_{1} \mathbf{b}_{1}+p_{2} \mathbf{b}_{2}, \quad s .
$$

Определим гамильтонову структуру рассмотренной динамической системы. Для этого необходимо задать фазовое пространство $\mathcal{H}$, пуассонову структуру (которая может быть вырожденной), гамильтониан $H$, а также систему первичных связей. Пусть указанные базовые объекты заданы следующим образом:

1) переменные, параметризующие фазовое пространство $\mathcal{H}$ :

$$
\left(\rho(\xi), \bar{\rho}(\xi) ; \quad q_{1}, q_{2} ; \quad p_{1}, p_{2} ; \quad s\right)
$$

4) В работе [8], где ступень иерархии $n=2$ рассматривалась как редукция релятивистской модели, полагалось $\mathcal{E}_{0}=m_{0} c^{2}$. 
2) скобки Пуассона

$$
\begin{aligned}
\{\rho(\xi), \bar{\rho}(\eta)\} & =\frac{1}{\alpha_{0} \sqrt{m_{0} \mathcal{E}_{0}}}\left[\delta^{\prime}(\xi-\eta)+\rho(\xi) \bar{\rho}(\eta) \varepsilon(\xi-\eta)\right], \\
\{\rho(\xi), \rho(\eta)\} & =-\frac{1}{\alpha_{0} \sqrt{m_{0} \mathcal{E}_{0}}} \rho(\xi) \rho(\eta) \varepsilon(\xi-\eta), \\
\left\{p_{l}, q_{j}\right\} & =m_{0} \delta_{l j}, \quad l, j=1,2
\end{aligned}
$$

(остальные скобки обращаются в нуль);

3) связь $\Phi\left[\rho(\xi) ; p_{1}, p_{2} ; s\right] \equiv \alpha_{0}^{2} \mathcal{P}^{2} f_{s}^{2}[\rho]-s^{2} \boldsymbol{f}^{2}[\rho] \approx 0$ (см. (25));

4) гамильтониан

$$
H=\frac{\mathcal{P}^{2}}{2 m_{0}}+\mathcal{E}_{0}(1+h[\rho])+l\left(\xi^{0}\right) \Phi,
$$

где функция $l$ - множитель Лагранжа.

Определенную таким образом гамильтонову динамическую систему будем называть "вихревая квазичастица на плоскости": такое название мотивировано рассмотренным выше соответствием данной системы бесконечной вихревой нити в приближении локальной индукции. Прокомментируем сделанное определение.

1. Теория содержит три размерные константы: $m_{0}, \alpha_{0}$ и $\mathcal{E}_{0}$ (либо $t_{0}$ ), задающие масштабы массы, длины и энергии соответственно. Роль времени в теории играет величина $t=t_{0} \tau$, так что для любого функционала $D=D[\rho ; p, q, s]$ в силу сделанных определений имеем

$$
\frac{\partial D}{\partial t}=\{D, H\}
$$

2. Скобки дискретных переменных $p_{i}, q_{i}$, а также $s$ (аннулятора) мотивированы структурой алгебры $\widetilde{\mathcal{G}}_{2}$, с учетом вида оператора Казимира $\widehat{C}_{2}$ (см. выше). Обратим внимание на работы [17], [18], в которых для описания энионов использовалось двухпараметрическое расширение группы Галилея, причем один параметр, как обычно, связывался с массой $m_{0}$, а второй - с внутренним моментом.

3. Формулы $(28),(29)$ для функций $\rho(\xi)$ и $\bar{\rho}(\xi)$ определяют пуассонову структуру $\{\cdot, \cdot\}_{1}$ в иерархии пуассоновых структур $\{\cdot, \cdot\}_{n}$ нелинейного уравнения Шредингера [11]. Заметим, что скобки (28) и (29) могут быть записаны локально в координатах $j_{a}(\xi) \equiv \operatorname{tr}\left[Q(\xi) \boldsymbol{\sigma}_{\boldsymbol{a}}\right]$ введением диагональной коэффициентной функции $j_{3}(\xi)$.

4. Условием, отбирающим функционалы $D$, допустимые для постулированных скобок, является требование $\{D, \operatorname{tr} \mathfrak{M}\}_{1}=0$ [11]. Это означает, что равенство (4) фактически представляет собой не связь, а условие отбора симплектических листов в фазовом пространстве. В настоящей работе мы не будем детально рассматривать эти вопросы.

5. Соответствие построенной гамильтоновой динамической системы и бесконечной вихревой нити имеет место только в интервале $0<\Gamma_{\varrho}<\infty$. При выполнении данного условия формулы (18) и (22) показывают, что из тождества $\rho(\xi) \equiv 0$ однозначно следуют равенства $\mathcal{P}=0$ и $s=0$. Мы не будем вводить такие ограничения на координаты фазового пространства $\mathcal{H}$ : неравенства $\mathcal{P} \neq 0$ и (или) $s \neq 0$ допустимы в случае выполнения тождества $\rho \equiv 0$, так же, как равенства $\mathcal{P}=0$ и $s=0$ возможны для функции $\rho \neq 0$. Это позволяет, например, рассматривать такой естественный (с точки зрения заявленной группы симметрии) объект, как свободную 
бесструктурную частицу на плоскости с собственным моментом $s$ и импульсом $\mathcal{P}$. В рамках представленной в настоящей работе интерпретации такую частицу можно отождествить с бесконечной прямолинейной вихревой нитью, движущейся в простанстве $E_{3}$ с постоянной скоростью $\mathcal{P} / m_{0}$.

Отметим, что при фиксированных внутренних степенях свободы $\rho(\xi)$ имеем

$$
s^{2} \propto \mathcal{E}+\text { const }
$$

что уже на классическом уровне дает представление о "траекториях" Редже в рассматриваемой модели.

\section{5. ЗАКЛЮЧЕНИЕ}

Обсудим кратко возможную схему квантования модели. Структура пространства $\mathcal{H}$ делает возможным выбрать гильбертово пространство состояний теории в виде

$$
\mathbf{H}=\int_{s} \mathbf{H}_{s}, \quad \mathbf{H}_{s}=\mathbf{H}_{2} \otimes \mathbf{H}_{\rho} .
$$

Здесь $\mathbf{H}_{2}$ - пространство квантовых состояний свободной нерелятивистской частицы на плоскости (т. е. пространство $L^{2}\left(\mathbb{R}_{2}\right)$ с необходимым оснащением), $\mathbf{H}_{\rho}$ - пространство квантовых состояний внутренних степеней свободы $\rho(\xi)$. Допустимые векторы состояния представимы в виде

$$
|\Psi\rangle=\int_{s} g(s)|s\rangle d s, \quad|s\rangle \in \mathbf{H}_{s}, \quad\left\langle s \mid s^{\prime}\right\rangle \propto \delta\left(s-s^{\prime}\right), \quad \int_{s}|g(s)|^{2} d s<\infty .
$$

Связь (25) приводит к уравнениям, определяющим допустимые векторы в каждом пространстве $\mathbf{H}_{s}$ :

$$
\left(s^{2} \hat{I} \otimes \widehat{\mathrm{P}}^{2}-\alpha_{0}^{2} \widehat{\mathcal{P}}^{2} \otimes \widehat{\mathrm{S}}^{2}\right)|s\rangle=0, \quad|s\rangle \in \mathbf{H}_{s},
$$

где единичный оператор $\hat{I}$ и оператор $\widehat{\mathcal{P}}^{2}=-\hbar^{2} \Delta$ действуют в пространстве $\mathbf{H}_{2}$, а операторы $\widehat{\mathrm{P}}^{2}$ и $\widehat{\mathrm{S}}^{2}$, действующие в пространстве $\mathbf{H}_{\rho}$, получены квантованием классических выражений $\boldsymbol{f}^{2}[\rho]$ и $f_{s}^{2}[\rho]$ соответственно. Предположим, что спектральная задача

$$
\widehat{\mathrm{S}}^{2}\left|\varphi_{n}\right\rangle=\lambda_{n}^{2} \widehat{\mathrm{P}}^{2}\left|\varphi_{n}\right\rangle
$$

в пространстве $\mathbf{H}_{\rho}$ имеет собственные векторы $\left|\varphi_{n}\right\rangle$ и собственные числа $\lambda_{n}^{2}>0$. Тогда общим решением уравнения (32) при каждом $s$ являются "запутанные" состояния

$$
|s\rangle=\sum_{n} a_{n}\left|\psi_{s, n}\right\rangle\left|\varphi_{n}\right\rangle
$$

где векторы $\left|\psi_{s, n}\right\rangle \in \mathbf{H}_{2}$ в координатном представлении суть

$$
\psi_{s, n}(\mathbf{r}) \equiv\left\langle\mathbf{r} \mid \psi_{s, n}\right\rangle=\sum_{l} c_{l} J_{l}\left(k_{s, n} r\right) e^{i l \phi}, \quad \mathbf{r}:(r \cos \phi, r \sin \phi)
$$

Здесь $l$ - угловой момент, $J_{l}$ - соответствующая функция Бесселя, $k_{s, n}=|s| / \lambda_{n} \alpha_{0} \hbar$. При этом спин $s$ принимает произвольные ненулевые значения. Состояния с $s=0$ 
существуют, если спектральная задача (33) имеет нетривиальные решения при $\lambda_{n}=0$.

Понятие квантовой частицы на бесконечной плоскости $E_{2}$ является, конечно, идеализированным, так что представляется естественным поставить те или иные граничные условия на функцию $\psi_{s, n}(\mathbf{r})$. В этом случае теория допускает только фиксированные (вообще говоря, дробные) значения спина $s$. Рассмотрим простейшие случаи.

1. Квазичастица с угловым моментом $l$ сосредоточена в круге радиуса $R_{0}$. Граничное условие $\psi_{s, n}\left(R_{0}, \phi\right)=0$ сразу приводит к выражению

$$
|s|=\hbar \lambda_{n} \zeta_{l}^{(m)}\left(\frac{\alpha_{0}}{R_{0}}\right), \quad n, m=1,2, \ldots,
$$

где $\zeta_{l}^{(m)}-m$-й ноль функции Бесселя $J_{l}$.

2. Квазичастица сосредоточена в пределах квадрата со стороной $R_{0}$. Аналогично находим

$$
|s|=\pi \hbar \lambda_{n} \sqrt{m_{1}^{2}+m_{2}^{2}}\left(\frac{\alpha_{0}}{R_{0}}\right), \quad n, m_{1}, m_{2}=1,2, \ldots
$$

Приведенные примеры, как и общие формулы (32) и (33), демонстрируют, что допустимые значения спина в предложенной модели определяются тремя факторами: 1) собственными числами спектральной задачи (33); 2) собственными числами оператора Лапласа для области, в которой может находиться квазичастица; 3) масштабным фактором $\alpha_{0} / R_{0}$, где $R_{0}$ - характерный размер указанной области. Параметр $\alpha_{0}$ в теории играет роль характерного размера квазичастицы: такое отождествление становится естественным, если записать выражение для проекции эволюционирующей кривой (2) на плоскость переменных $\boldsymbol{q}$ и $\mathbf{p}$. Что касается общего пространства квантовых состояний, то в его определении (31) при наличии граничных условий интеграл должен быть заменен на сумму по допустимым (для конкретной модели) значениям спина, с соответствующим изменением условий нормировки на векторы $|s\rangle$. Конечно, детальному исследованию элементов представленной схемы должна быть посвящена отдельная работа.

В заключение заметим, что предложенная схема квантования была реализована автором подробно в несколько иной модели (конечная струна на плоскости) в работе [19].

\section{Список литературы}

[1] M. Levin, X.-G. Wen, Rev. Modern Phys., 77:3 (2005), 871-879, arXiv: cond-mat/0407140.

[2] W. Thomson, Phil. Mag., 34 (1867), 15-24.

[3] K. Моффатт, Нелинейная динам., 2:4 (2006), 401-410.

[4] F. Wilczek, Phys. Rev. Lett., 48:17 (1982), 1144-1146.

[5] G. Moore, Nucl. Phys. B., 360:2-3 (1991), 362-396.

[6] А.П. Протогенов, УФН, 162:7 (1992), 1-80.

[7] M. G. Alford, F. Wilczek, Phys. Rev. Lett., 62:10 (1989), 1071-1074.

[8] С. В. Талалов, ТMФ, 165:2 (2010), 329-340.

[9] С. В. Талалов, ТМФ, 152:3 (2007), 430-439.

[10] П. П. Кулиш, А. Г. Рейман, Зап. научн. сем. ЛОМИ, 77 (1978), 134-147. 
[11] Л. А. Тахтаджян, Л. Д. Фаддеев, Гамильтонов подход в теории солитонов, Наука, М., 1986.

[12] Ф. Дж. Сэффмен, Динамика вихрей, Научный мир, М., 2000.

[13] С. В. Алексеенко, П. А. Куйбин, В. Л. Окулов, Введение в теорию концентрированных вихрей, Ин-т теплофизики СО РАН, Новосибирск, 2003.

[14] Дж. Бэтчелор, Введение в динамику жидкости, Мир, М., 1973.

[15] Н.Я. Виленкин, Специальные функции и теория представлений групп, Наука, М., 1965.

[16] В. И. Фущич, А. Г. Никитин, Симметрия уравнений квантовой механики, Наука, М., 1990.

[17] R. Jackiw, V.P. Nair, Phys. Lett. B, 480:1-2 (2000), 237-238, arXiv: hep-th/0003130.

[18] J. Negro, M. A. del Olmo, J. Tosiek, J. Math. Phys., 47:3 (2006), 033508, 19 pp., arXiv: math-ph/0512007.

[19] S. V. Talalov, Internat. J. Modern Phys. A., 26:16 (2011), 2757-2772, arXiv: 1105.0743.

Поступила в редакцию 9.01.2013, после доработки 17.03.2013 